\title{
DE ORGANIZACIONES INDÍGENAS A PARTIDOS ÉTNICOS: NUEVAS TENDENCIAS EN LAS LUCHAS INDIAS en AmÉRICA LATINA
}

\author{
Araceli Burguete Cal y Mayor
}

Resumen: En esta colaboración se examina las recientes transformaciones de la política electoral en países de América del Sur, con énfasis en Ecuador y Bolivia, en virtud de la creciente incorporación de organizaciones indígenas en la lucha por el poder político, lo que ha dado lugar a un nuevo tipo de partidos: los partidos étnicos. Con este trasfondo, reviso los posicionamientos de los principales paradigmas teóricos en el estudio de los movimientos sociales, frente a la relación entre los partidos políticos y los movimientos sociales. Y exploro pistas que puedan dar cuenta de los escenarios de construcción de "partidos étnicos" en México.

Palabras claves: organizaciones indígenas, movimientos sociales, partidos políticos y partidos étnicos.

Enviado a dictamen: 10 de febero de 2007. Aprobación: 16 de agosto de 2007.

Araceli Burguete Cal y Mayor, doctora en Antropología social, investigadora del Ciesas-Sureste.
Abstract: In this collaboration it is examined the recent transformations of the electoral policy in countries of South America, with emphasis in Ecuador and Bolivia, by virtue of the increasing incorporation of indigenous organizations in the fight by the political power, which has given rise to a new type of parties: the ethnic parties. With this trasfondo, I review the positionings of the main theoretical paradigms in the study of the social movements, as opposed to the relation between the political parties and the social movements. And I explore tracks that can give account of the scenes of construction of "ethnic parties" in Mexico.

Key words: Indigenous organizations, social movements, political parties and ethnic parties.

\section{Partidos políticos indígenas y partidos étnicos en América Latina}

E "ejemplo Evo" parece estar alentando a los actores sociales indígenas a entrar en el terreno de la lucha político electoral. El triunfo de Evo Morales en las elecciones a la presidencia de la República en Bolivia, en diciembre de 2005, parece tener algunas repercusiones en el movimiento indígena continental. La consigna "De la resistencia al poder", lema de la convocatoria emitida en encuentros continentales indígenas celebrados en Bolivia en el año 2006, y en Guatemala, en el año 2007, da cuenta del impacto simbólico que tal 
triunfo tiene para segmentos importantes del movimiento indígena. ${ }^{1}$

Desde hace una década, en países del cono sur, dio inicio la formación de partidos políticos que se han autodescrito como indígenas. La mayoría de ellos son resultado de diversos procesos de reconfiguración vividos por las organizaciones indígenas, las que habrían de convertirse en partidos políticos; transformación que Van Cott $(2003,2005)$ ha nombrado como "partidos étnicos". En la última década, las organizaciones indígenas no se han limitado a reclamar derechos, sino que se han organizado para disputar poder, representación y proyecto político. Y en algunos casos, como Ecuador y Bolivia, han sido los actores políticos más dinámicos al conducir los procesos nacionales más significativos de la historia contemporánea de esos países, encabezando las luchas por la defensa de los recursos estratégicos como el agua, el gas y el petróleo, al mismo tiempo, han competido en liderazgo con los partidos políticos convencionales. Pero, ¿es posible la multiplicación de los partidos étnicos en regiones de América Latina distintas a las del cono sur? ¿Y si ha sido así, qué condiciones incentivaron su constitución? ¿Basta con que una organización indígena se declare constituida como partido político para que éste pueda lograr éxitos electorales? Esta colaboración intenta explorar algunas respuestas a estas preguntas.

Una mirada rápida a algunos eventos acontecidos en los primeros años del nuevo siglo, da cuenta de estos intentos. En el primer semestre de 2007 se dieron a conocer iniciativas de formación de "partidos políticos indígenas" en distintos países de América Latina. Se comenzó en Guatemala. El anuncio de la candidatura de Rigoberta Menchú a la presidencia de la República en Guatemala y la posibilidad de crear un partido político indígena fue un tema ampliamente debatido en los medios de comunicación durante todo el primer semestre de $2007 .^{2}$

En la primera semana de mayo se anunció en Perú la creación del Movimiento al Socialismo Andino-Amazónico (emulando al Movimiento al Socialismo, que llevó al poder a Evo Morales), en el marco de la celebración del "Primer Congreso Político del Movimiento Indígena del Perú". Este nuevo organismo se constituía, a decir de sus organizadores, como "un instrumento político" para la "culminación de las tareas de la descolonización y la conquista de la soberanía y la dignidad de los pueblos del Perú". ${ }^{3}$ Dos semanas después, la provincia de Neuquén en Argentina, fue el escenario de convocatoria del "Partido Pueblo Originario Mapuche (PPOM)", como una iniciativa de los mapuches de ese país de contar con institutos políticos que les permitieran ganar los espacios de gobernador y vicegobernador en la provincia de Neuquén. Este nuevo partido nacía, a decir de sus convocantes, como una respuesta de rechazo a lo que caracterizaban como una "situación de marginación política desde los partidos que están en el poder". El PPOM se asumía como “(...) el primer intento mapuche en Neuquén por crear una fuerza política propia, abierta a la participación electoral, ya sea solos o en alianza con sectores opositores (...)". ${ }^{4}$

Antes, en el segundo semestre de 2005, el reconocido líder mapuche Aucán Huilcamán de la organización indígena "Consejo de Todas las Tierras", en Chile, intentó competir como candidato a la presidencia de la República, apoyado por la Red Indígena y Popular, pero diversos obstáculos de la legislación electoral de ese país impidieron su registro. La negativa causó una gran polémica internacional, lo que condujo incluso a un comentario diplomático desde la Unión Europea y a que diversas personalidades del mundo se pronunciaran a su favor. ${ }^{5}$ Mientras que en Ecuador, el también reconocido líder de la organización indígena Confederación Nacional Indígena del Ecuador (CONAIE), Lucio Macas, fue candidato, en 2006, a la presidencia de la República.

Al observar el surgimiento de partidos políticos indígenas, que disputan los espacios que tradicionalmente ocupaban los partidos políticos convencionales en países de América del Sur, Van Cott percibe la novedad de la presencia de un nuevo actor: "los partidos étnicos". La autora define al "partido étnico" 
DE ORGANIZACIONES INDÍGENAS A PARTIDOS

ÉTNICOS: NUEVAS TENDENCIAS EN LAS LUCHAS

[...] como una organización autorizada para participar en las elecciones locales o nacionales, cuyos líderes y miembros en su mayoría se identifican a sí mismos como parte de un grupo étnico no gobernante y cuya plataforma electoral incluye demandas y programas de naturaleza étnica o cultural [...] Mi definición incluye a entidades que se llaman a sí mismas 'movimientos políticos' para distanciarse retóricamente de las connotaciones negativas asociadas con los partidos políticos en sus sociedades, pero que de cualquier otra manera cumplen la definición (2003: 27).

La hipótesis de la autora es que los cambios institucionales asociados con políticas de reconocimiento de derechos indígenas, junto con reformas electorales que ampliaron la participación ciudadana, favorecieron la creación de "partidos étnicos", con probabilidades de alcanzar triunfos. Este cambio ha ejercido una fuerte presión en el sistema de partidos, lo que ha obligado a la "metamorfosis" de éste. Antes del triunfo de Evo Morales, un tema álgido en la opinión pública era el devenir de los partidos políticos por la crisis que enfrentaban. A decir de Mayorga, los bolivianos se interrogaban sobre si los movimientos sociales como el "Instrumento Político por la Soberanía de los Pueblos (IPSP)", que había fundado Evo Morales, antes de obtener el registro por el Movimiento al Socialismo (MAS), estaban llamados a sustituir y eventualmente a eliminar a los partidos. El autor concluye que los partidos sobrevivieron, pero fueron necesarias diversas mutaciones. Lo dice con las siguientes palabras:

Contrario a lo que se esperaba, el sistema de partidos boliviano no se ha derrumbado ni ha llegado a su fin. El cambio notable ha sido fundamentalmente la desaparición y declinación de algunos de los partidos y el surgimiento de otros, lo cual equivale a una metamorfosis pero no a una disolución del sistema de partidos como tal (2003: 103).
La experiencia sobre la formación del IPSP, en Bolivia, tiene semejanzas con la de Ecuador, en la que se observa esta tendencia de reconfiguración de las organizaciones indígenas en su tránsito hacia partidos políticos, las que de manera creciente se han incorporado a luchas que tienen por objeto el poder político, en distintos ámbitos: local, regional y nacional. Hidalgo (2002) refiere que el contexto que facilitó la irrupción de la CONAIE en el terreno de la política electoral estuvo precedido por una "quiebra de los partidos políticos". Al mismo tiempo, los (viejos) movimientos sociales, como las organizaciones sindicales, entraron en un proceso de deterioro como consecuencia de las transformaciones en la organización productiva. En un contexto de crisis económica y política, provocado por la corrupción, la dolarización de la economía y el crecimiento de la pobreza, las organizaciones sociales ganaron presencia nacional, y ofrecieron alternativas a las formas existentes de hacer política (Burguete, 2000a).

F. García (2005) indica que éstos y otros factores -como la formación de nuevos liderazgos y la decepción que experimentó el movimiento indígena respecto al sistema político, que había omitido dar respuesta al principal reclamo de aquél de modificar el Estado uninacional, para dar paso a un Estado plurinacional- condujeron a la CONAIE a decidir incorporar en su agenda la lucha por el poder político y transitar "de un movimiento social a un partido político", al dar nacimiento al Movimiento de Unidad Plurinacional Pachakutik (MUPP). A decir de P. Dávalos (2003), la CONAIE dio nacimiento al MUPP como una estrategia de lucha que permitiera disponer de un "instrumento político" para acceder al poder del Estado y construir un "poder alternativo" (Botero, 1998).

Favorecido por el entorno nacional, el movimiento Pachakutik fue configurándose como un "partido étnico", que ofreció alternativas políticas para todos los ecuatorianos, y se extendió más allá de la agenda indígena. Adquirió y amplió su presencia política nacional, en tanto fue capaz de configurarse como un 
movimiento multiétnico, multiclasista y multisectorial (Dávalos, 2003; Barrera, 2001; Guerrero, 1996; Hidalgo, 2002). El Pachakutik ha sido exitoso en el terreno de los gobiernos locales y provinciales, pero ha enfrentado dificultades y ha tenido que pagar costos muy altos en los últimos tres años, como resultado de la fallida alianza que realizó con el coronel Lucio Gutiérrez, a quien llevó a la presidencia de la República de ese país, un hecho con consecuencias graves para el movimiento social, por las tensiones vividas con los líderes de la CONAIE. Pese a estas dificultades, el movimiento indígena ecuatoriano ha logrado modificar la vida política nacional (Carvajal, 2004). Máiz (2005) evalúa que la CONAIE-Pachakutik ha proporcionado alientos refrescantes a la democracia y a la sociedad ecuatorianas.

Aunque en su definición de "partido étnico", Van Cott (2003) no distingue éste de la acepción de "partido político indígena", e incorpora este último bajo la misma denominación. En contextos como Bolivia, la distinción entre uno y otro parece tener sentido. Autores como R. Calla (1993) llaman la atención sobre la importancia de distinguir entre "movimiento indígena" y "movimiento de identificación étnica". El primero se refiere a participantes cuyos miembros se adscriben a una identidad étnica indígena, mientras que el "movimiento de identificación étnica" integra por igual a indígenas y no indígenas en un proyecto político. La "identificación étnica”, precisa, “...alude a procesos complejos de solidaridades y opciones ideológicas conscientes" de adhesión a proyectos políticos de base étnica:

[...] una identificación étnica en cuanto base de una acción política en la que confluyen tanto sujetos externosal mundo aymara, quechuay otros autodenominados 'pueblos indígenas'u 'originarios' como sujetos provenientes desde dentro de ese universo étnico (1993: 61).

Esta configuración remite a las historias nacionales y a vínculos históricos entre etnia y clase. Autores como Mamani (2006), Patzi (2005), Albó (1996), Rivera (2003),
García Linera (2005), Quispe (2006) y Calla (1993), entre otros, han documentado viejas relaciones entre etniaclase, movimiento indígena y lucha sindical, en una compleja amalgama, en la Confederación Sindical Única de Trabajadores Campesinos de Bolivia, (CSUTCB), articulada a partir de la identidad obrero-campesina. Más recientemente se han fundado "partidos cocaleros" (productores de la hoja de coca) de configuración pluriétnica, en la región de El Chapare caracterizada por ser una zona de colonización con un perfil multiétnico. Las múltiples pertenencias identitarias han cultivado una cultura política indígena de alianzas con los campesinos y los obreros del país, y más recientemente con diversos sectores de la sociedad nacional, en la defensa común de los principales recursos estratégicos del país, como el agua, el gas y el petróleo.

Con esa densa amalgama étnico-política como antecedente, fue posible la configuración de un "movimiento de identificación étnica" que involucra a miles de ciudadanos, indígenas y no indígenas. Así, para comprender la formación de un "partido étnico" y el triunfo de Evo Morales, resulta útil la distinción que realiza R. Calla (1993) entre "movimiento étnico" y "movimiento indígena”. Por la amplia votación a favor de Morales (más del $50 \%$ de los sufragios) en las elecciones presidenciales de 2005, podemos inferir que los votos se produjeron como resultado de un movimiento de "identificación étnica", que fue más allá del "voto indígena". En este orden de ideas, "partido étnico" en Bolivia, no sería lo mismo que "partido indígena". El primero es multiétnico, multiclasista y multisectorial. Aunque hay que recordar que para que los partidos étnicos fueran posible en Bolivia, hubo desde la década de los setenta, movimientos indígenas que pugnaban por la restauración del Tawantinsuyu y formaron "partidos indígenas", como el partido Tupak Katari. Importa destacar que, en este caso, el partido étnico no supone ruptura con el partido indígena, sino la reconfiguración del segundo con su irrupción en el escenario nacional. Los partidos étnicos que actúan en el ámbito nacional suelen alimentarse, desde abajo, de 
DE ORGANIZACIONES INDÍGENAS A PARTIDOS

partidos indígenas; es decir, de partidos que en la escala municipal y regional pujan por la representación política indígena, aunque en el contexto nacional puedan realizar alianzas con diversos grupos y sectores (Albó, 1996; Calla, 1993; Rivera, 2003).

El nacimiento de los partidos de "configuración étnica" tiene un origen más reciente. Van Cott (2003) documentó la constitución del "Instrumento Político por la Soberanía de los Pueblos (IPSP)” creado como un mecanismo electoral por los cocaleros de la región de El Chapare:

La idea de formar un vehículo electoral fue discutida en conferencias previas de la CSUTCB. Esta organización se convirtió en una opción viable impulsada principalmente por los cocaleros tras la creación de oficinas municipales, elegidas a lo largo del país, apoyando a aquellos que argumentaban que el movimiento campesino podía transformar el Estado boliviano ocupando espacios de poder ya establecidos (2003: 42).

Algunos líderes de la CSUTCB argumentaron que tal paso era necesario para ganar "el espacio político democrático". El nuevo partido, con una figura de "movimiento", comenzó a ganar las elecciones municipales y, junto con ello, se inició el progresivo deterioro de los partidos políticos tradicionales que perdieron sus clientes seguros. En 1997, el partido cocalero ganó cuatro de las nuevas curules uninominales del Congreso, favorecido por nuevas reformas municipales y electorales. En esa ocasión, el líder cocalero Evo Morales obtuvo una copiosa votación en su distrito, así, fue electo diputado.

Por este tipo de articulación, el "partido étnico", en Bolivia, tiene detrás la construcción de capacidades, liderazgos y legitimidad para representar a distintos sectores de la sociedad y no únicamente a los indígenas. Por tal motivo, una novedad adicional de esa articulación movimiento social-partido político es que una vez que han ganado en las urnas, las organizaciones sociales que lo integran se incorporan al gobierno como parte de una coalición gobernante (con distinto éxito: más favorable para el movimiento indígena de Bolivia, que para el de Ecuador) que accede al poder nacional. La coalición gobernante puede gozar de legitimidad y, una base social suficientemente sólida, que le permita impulsar políticas "populares", e implementar políticas públicas que buscan romper el llamado "Consenso de Washington", ${ }^{6}$ yendo más allá de las políticas neoliberales con base en medidas nacionalistas.

Como ha sido el caso de Bolivia, la fórmula movimientos sociales y partidos políticos parece marcar una tendencia más amplia, que va más allá de las alianzas con las organizaciones indígenas. Los triunfos electorales, en el año 2006, en países de América Latina, de candidatos respaldados por alianzas, movimientos y partidos políticos de orientación de izquierda, ponen sobre la mesa la pertinencia de reflexionar sobre estas nuevas formas de acción colectiva. Las victorias electorales de Hugo Chávez (Venezuela), Luiz Inácio Lula Da Silva (Brasil, con el apoyo del Movimiento de Los Sin Tierra, en Brasil, en alianza con el Partido del Trabajo), Rafael Correa (Ecuador) y Daniel Ortega (Nicaragua) dan señales de innovaciones en la política de la región (Touraine, 2006; Cheresky, 2006).

A primera vista, estos resultados electorales tienen por lo menos cuatro consecuencias: i) aportar nuevas bases que trasciendan el mero mecanismo formal para la elección de gobernantes, con tal de relegitimar la democracia electoral como un espacio posible para el cambio político; ii) llevar al poder, mediante la vía electoral, a gobiernos nacionalistas que buscan enfrentar la pobreza de la región, saliéndose del guión dictado desde Washington y recuperar la soberanía y los recursos estratégicos del país; iii) introducir innovaciones en el campo de la política al diseñar un modelo de articulación que ha tenido resultados exitosos, y iv) transformar el sistema de partidos y a los partidos políticos mismos, que están obligados a modificarse, tanto en su integración como en su programa y política de alianzas. 
En resumen, en esta coyuntura, por primera vez las organizaciones indígenas y sus liderazgos tienen la oportunidad de superar su condición de clientes seguros de los partidos políticos convencionales (Ruiz y Burguete, 2001), al mismo tiempo que los movimientos sociales indígenas comienzan a tener un papel relevante en el surgimiento de estas nuevas formas de hacer política en América Latina (Máiz, 2005; Martí, 2006; Van Cott, 2004; Mirza, 2006; Ruiz, 2003; Somuano, 2007). Para asistir a estos escenarios de la política en el nuevo milenio, las organizaciones indígenas se han reconfigurado y su ejemplo alienta a liderazgos, en otros países, a disputar el poder político nacional, regional y local.

\section{Movimientos sociales y partidos políticos: una breve nota teórica}

La literatura sobre movimientos sociales es abundante. Pese a su diversidad, la mayoría de los autores que se han ocupado de su clasificación identifican dos paradigmas colocados uno frente al otro: el "paradigma de la identidad" y el "paradigma estratégico" o de la "movilización de recursos" (Ramos, 1997; Cohen, 1985; Rubio, 2004). Todos ellos, también, ignoran el aporte de las investigaciones realizadas desde América Latina, las que aquí identifico dentro del "paradigma emancipatorio", que enfatiza la acción colectiva de los movimientos sociales en las luchas por la (s) democracia (s)en América Latina (Flórez, 2005). Desde cada paradigma, los vínculos entre movimientos sociales y partidos políticos se perciben de manera diferente. Brevemente, acerquémonos a estos enfoques.

\section{Nuevos Movimientos Sociales (NMS), el paradig- ma de la identidad y los partidos políticos}

Melucci, uno de los teóricos europeos más reconocidos en el estudio de los Nuevos Movimientos Sociales (NMS), afirma que en el último tercio del siglo XX se asiste a la aparición de un nuevo paradigma de la acción colectiva lo que da origen a los NMS. Éstos se caracterizan porque apelan a la solidaridad, manifiestan un conflicto y conllevan una ruptura de los límites de compatibilidad del sistema dentro del cual la acción tiene lugar. A este enfoque se le denomina "paradigma de la identidad". Estos movimientos expresan sus reclamos mediante formas no institucionales de acción colectiva en sistemas complejos. Desde su perspectiva, las formas organizativas de las instituciones políticas tradicionales, incluidas las procedentes de una tradición de izquierda, son en sí mismas inadecuadas para representar las nuevas demandas colectivas. El espacio social en el que esos movimientos actúan es el de la vida cotidiana, un área diferenciada del sistema y han dejado de coincidir con las formas tradicionales de organización de la solidaridad y con los canales convencionales de representación política. Desde esta perspectiva, los NMS, a diferencia de los "viejos movimientos sociales", son luchas ciudadanas que se sitúan en el terreno del "antagonismo simbólico" y combinan la lucha por las garantías civiles y democráticas, por el logro de formas de participación que buscarían traducirse en nuevas reglas y nuevos derechos (Melucci, 1999: 9-19). Por ese núcleo antagonista, los NMS se distancian de los partidos y de las formas estatales de hacer política, de lo que resulta una tensión entre los NMS y los partidos políticos.

De esta tensión también se ha ocupado Claus Offe. En un contexto de "crisis del sistema" y del Estado de bienestar y de la pérdida de legitimidad del sistema político vigente, los partidos políticos se debilitan. Aunque el autor admite que los movimientos sociales no tienen como objetivo, al menos situado en primer plano, la lucha parlamentaria y el ejercicio eventual del poder, en tanto los partidos políticos disminuyen su capacidad de representación y canalización de las demandas ciudadanas, dichos paridos tienden a ser desplazados por los NMS, que se convierten en vehículos para canalizar los reclamos sociales (1998: 31).

Oñate (2005), por su parte, al profundizar en la distinción entre NMS y partidos políticos, identifica que las diferencias no se reducen a cuestiones de forma (a las 
DE ORGANIZACIONES INDÍGENAS A PARTIDOS

ÉTNICOS: NUEVAS TENDENCIAS EN LAS LUCHAS

INDIAS EN AMÉRICA LATINA

Abierta

formas de hacer política) sino que son asuntos de fondo: de paradigmas políticos. Distingue entre la "vieja política" de los partidos, y la "nueva política" de los movimientos sociales, una de cuyas diferencias se patentiza en las modificaciones en la agenda. Se pasa de temas vinculados al bienestar material (progreso, desarrollo, crecimiento, etcétera) a otros nuevos ("inmateriales") como ecología, identidad, solidaridad, autonomía, autoestima, entre otros. Oñate destaca que la emancipación por la que luchan los movimientos sociales no es política, sino ante todo personal, social y cultural; con ello, la agenda de los NMS representa, de alguna forma, la afirmación de la subjetividad frente a la ciudadanía. Menciona también cambios en el estilo de actuación. La negociación y el pacto que caracteriza a la "vieja política" que encarnan los partidos políticos tradicionales y los "viejos movimientos sociales", como los sindicatos, son abandonados, sustituidos por el radicalismo y la innegociabilidad como principio. Desde esta perspectiva teórica, los NMS y los partidos políticos actúan en terrenos antagónicos y su relación se presume como poco probable y poco deseable.

\section{Movimientos sociales y partidos políticos en el paradigma emancipatorio en América Latina}

Boaventura de Sousa Santos (2001) toma una posición crítica frente a la perspectiva del "paradigma de la identidad". Cuestiona la afirmación de la preeminencia de la subjetividad frente a la ciudadanía, enfoque que descarta las luchas por la democracia representativa, enfatizando en la democracia participativa, rechazando la lucha por el poder político. Santos cuestiona que la diversidad de movimientos que se despliegan en los países de América Latina, y los múltiples sentidos de la acción colectiva, pueda ser explicada en su totalidad por una teoría unitaria. Además, no ignora que las teorías sobre los NMS reflexionan principalmente sobre la irrupción de estos movimientos en los países con un capitalismo avanzado ("sociedades complejas"), que transitan de valores materialistas a "valores postmaterialistas".
Llama la atención sobre las diferencias significativas en términos de objetivos, de ideología y de base social entre los NMS de los países centrales y los de América Latina, entre los "valores postmaterialistas" y las necesidades básicas; entre la alineación y el hambre, las críticas al consumo en sociedades que carecen de la satisfacción de las necesidades básicas. Desde su punto de vista, la lucha por la ciudadanía es lo que hace que los movimientos sociales en América Latina mantengan un "eslabón" que los une con "viejos movimientos sociales", toda vez que, a diferencia de los países del centro, estos derechos son cuentas pendientes para su realización. También lo es el hecho que una parte significativa de los esfuerzos de los movimientos sociales en América Latina se haya canalizado a lograr la democracia, sin que ésta se agote en la forma representativa. Retoma los aportes de Fernando Calderón y Elizabeth Jelin, cuando afirman que en contraste con lo que sucede "en los países centrales", “...una de las características propias de América Latina es que no hay movimientos sociales puros o claramente definidos, dadas la multidimensionalidad, no solamente de las relaciones sociales sino también de los propios sentidos de la acción colectiva..." (2001: 180). De lo que resulta que "La novedad de los NMS no reside en el rechazo de la política sino, al contrario, en la ampliación de la política más allá del marco liberal de la distinción entre estado y sociedad civil" (2001: 181).

Como lo ilustran los casos de Bolivia y Ecuador, en América Latina, los viejos partidos políticos han sido rebasados por los movimientos sociales. Para Boron, esta situación de crisis no se limita a los partidos, sino que incluye al sistema de partidos, como resultado de la subordinación de la democracia al capitalismo y con ello la deslegitimación de la política. Esta situación "abrió" un espacio para que "la calle" adquiriera un renovado y acrecentado protagonismo en los procesos democráticos en la región, evidenciando el papel de los partidos como legitimadores de las políticas dictadas por el llamado "Consenso de Washington". Pero, a diferencia del "paradigma de la identidad", Boron no 
apuesta por la centralidad de los movimientos sociales como los principales actores de las luchas sociales (2005). Desde su perspectiva, los movimientos sociales tienen límites, propios de su "inmediatez espontaneísta", por lo que considera imprescindible la alianza de éstos con los partidos políticos. Un requisito previo es que los partidos políticos de izquierda, enfatiza el autor, asuman proyectos emancipatorios y articulen distintas formas de lucha, que no se limiten a la electoral. Se trata, propone, de construir una estrategia que "combine creativamente la calle con las urnas" (Boron, 2006).

Agregaríamos que, en esta lógica, los movimientos sociales ya no aceptarían relacionarse con los partidos políticos como clientes subordinados, sino sólo sobre la base de una nueva relación que presuma la integración de coaliciones gobernantes, de las que los liderazgos de las organizaciones sociales reclaman ser parte. Estrategia que, presumiblemente, tendría como objetivo no sólo la "toma del poder político", sino también "el poder del Estado", que permitiera la transformación de éste. Recordemos que al referirse a las alianzas entre los partidos políticos y los movimientos sociales en América Latina, González Casanova llamaba la atención sobre la importancia de distinguir entre la pugna política por lograr el "poder del gobierno" o "ganar el poder del Estado". Lograr esto último permitiría a los partidos y los movimientos impulsar procesos de emancipación social. Lo otro se reduce a puestos dentro de la estructura de gobierno o a cargos de representación popular, sin modificar la organización del Estado (1993: 21). Aún más, agregaría, el movimiento indígena no sólo se propone la "toma del poder del Estado" sino su refundación, tal y como se lo han planteado las organizaciones indígenas de Ecuador y Bolivia, que se han propuesto transitar de un "Estado monoétnico" a un "Estado plurinacional" (García Linera, 2005; Dávalos, 2003; García, s/f). Ésta parece ser la estrategia que algunas organizaciones indígenas se han planteado, como pudimos dar cuenta en la introducción de esta comunicación.

\section{Movimientos sociales y partidos políticos en el "Paradigma Estratégico"}

En el "Paradigma Estratégico" o de "Movilización de Recursos", se incluyen distintos enfoques teóricos. Habiendo tenido su origen en los Estados Unidos, todos éstos se caracterizan por rechazar consideraciones de carácter psicosocial. De las corrientes teóricas más importantes de las que este paradigma se ha alimentado, destacan tres: $i$ ) la Teoría de la Elección Racional, ii) la movilización por "gestión de recursos"y iii) el Enfoque del proceso político o la "Estructura de Oportunidades Políticas" (Rubio, 2004; Cohen, 1988; Jenkins, 1988). ${ }^{7}$ Esta última es la que resulta más pertinente al tema que aquí nos ocupa.

El enfoque de la "Estructura de Oportunidades Políticas" (EOP) se inscribe en las nuevas formulaciones del enfoque centrado en la movilización, organización y gestión de recursos. Eisinger acuñó el término, en 1973, para indicar el grado de apertura o cierre de un sistema político dado y su relación con la irrupción de los movimientos sociales. Desde esta perspectiva, los cambios producidos en el sistema político pueden ser percibidos como incentivos con los que los actores llevarían a cabo la acción colectiva. Tarrow, uno de sus teóricos más emblemáticos plantea que:

En los sistemas democráticos, las elecciones son eventos rutinarios, dominados habitualmente por los partidos constitucionales que aprueban las leyes necesarias para mantener su monopolio de represetación. Pero en los sistemas no democráticos, las nuevas posibilidades de acceso pueden desencadenar la acción colectiva [...] Cuanto más estrechos son los caminos de participación preexistentes, más probable resulta que cada nueva apertura produzca nuevas oportunidades (2004: 118).

Como es evidente en el planteamiento de Tarrow, el paradigma de la EOP presume una coexistencia entre 
DE ORGANIZACIONES INDÍGENAS A PARTIDOS

ÉTNICOS: NUEVAS TENDENCIAS EN LAS LUCHAS

INDIAS EN AMÉRICA LATINA

Abierta

movimientos sociales y partidos políticos y pondera la agencia de los actores para, estratégicamente, aprovechar las oportunidades políticas, cuando se presentan aperturas como resultado de las reformas en el sistema político. Desde este paradigma, la "percepción de oportunidad" contribuye a expandir los movimientos sociales. Éste es el enfoque al que se adhiere la investigación de Van Cott $(2003,2005)$. Desde su punto de vista, las políticas de reconocimiento de derechos indígenas, aunadas a la apertura que produjeron nuevos constituyentes y cambios en los sistemas políticos electorales, abrieron la oportunidad para la acción colectiva indígena, la que vio espacios favorables para entrar en el terreno electoral y disputar el poder del Estado. Dicha colectividad procedió, entonces, a reconfigurar sus organizaciones sociales para constituirlas en movimientos, instrumentos, partidos políticos, asistiendo a los campos electorales con alta probabilidades de éxito, capaces de desplazar a los partidos políticos convencionales.

Recurriendo a este mismo instrumental teóricometodológico, Máiz (2005) y Martí (2006, 2004) coinciden con otros autores como Bengoa (2000), Gros (2000), Díaz-Polanco y Sánchez (2002), quienes, desde otros paradigmas teóricos, evalúan que las luchas indígenas latinoamericanas no se limitan a demandas "posmaterialistas" ( pósitos de poder específicos, como por ejemplos todos aquellos que resultan del ejercicio del derecho a la libre determinación, autonomía y autogobierno. Además, con estas luchas, se reclama la vigencia de derechos ciudadanos hasta ahora no logrados, por lo que tienen que disputarlos frente al Estado-nación. Lo que supone, necesariamente, algún tipo de negociación de derechos "materiales". Desde estos enfoques, el movimiento indígena aspira a traducir su presencia en logros concretos de reconocimiento de derechos y de redistribución del poder, y no agota su lucha en lo meramente simbólico, en meras políticas de reconocimiento cultural. Así, la constitución de "partidos étnicos" en Ecuador y Bolivia, y los esfuerzos desplegados por organizaciones indígenas para constituirse como tales en otros países de la región, puede ser visto como una nueva estrategia desde el movimiento indígena de América Latina para la realización de esos derechos.

Sin embargo, conviene llamar la atención sobre las limitaciones de esta estrategia. Recordemos que la hipótesis de Van Cott (2003) enfatiza la importancia de los contextos nacionales en los que existe una coyuntura de reformas institucionales que incluyen reformas en el sistema electoral para facilitar la representación ciudadana, como condición necesaria. Estas condiciones no se han dado, por ejemplo, en Perú; por lo que, no obstante la vecindad con Bolivia y Ecuador y la supremacía demográfica indígena en ese país, los partidos étnicos no han irrumpido. Lo mismo podríamos decir para el caso de Guatemala. Pese a la importancia demográfica indígena y a los esfuerzos realizados por Rigoberta Menchú, no se logró constituir el anunciado partido étnico; por ello, ella tuvo que registrar su candidatura bajo las siglas de un partido político convencional para contender por la presidencia de la República, en $2007 .^{8}$

Así, aunque con la candidatura de Menchú se cumple la hipótesis de la nueva tendencia en el movimiento indígena a ocupar nuevos espacios en el poder político para que tales resultados sean exitosos, se requieren las otras variables mencionadas por Van Cott.

Dentro estos límites, ¿existen condiciones para la constitución de partidos étnicos en México?

\section{¿Partidos étnicos en México?: diversidad política indígena y la cuestión de las escalas}

De los resultados de investigación de campo obtenidos por Van Cott (2003) sobre el surgimiento de partidos étnicos en América del Sur, nos hemos referido hasta aquí únicamente a partidos étnicos de alcance nacional, en Ecuador y Bolivia. Pero la investigación realizada por dicha autora es más amplia, su universo de estudio incluye partidos políticos indígenas en la escala local, como los casos de Colombia, Venezuela y Argentina. En estos 
países la población indígena se encuentra regionalmente localizada. La concentración de la población en regiones, provincias o distritos constituye una fortaleza y una oportunidad, ya que en estos casos los partidos políticos sí tienen un propósito expreso de ganar representación política indígena. El perfil de estos partidos es, efectivamente, como un "partido político indígena", y no como "partido político étnico", según vimos arriba para el caso de los partidos étnicos de Ecuador y Bolivia.

En la escala regional, estos partidos se han constituido para la elección de representantes indígenas a los congresos locales y nacionales, además de participar en contiendas para elegir autoridades locales en los municipios y en los distritos y provincias regionales. Las organizaciones nacionales de Bolivia y Ecuador también participan en estas escalas. De hecho, su arribo a la escala nacional comenzó con éxito en este nivel de representación.

Esta distinción es importante ya que abre la posibilidad de indagar sobre "partidos políticos indígenas", en otras escalas, en realidades nacionales distintas a las del Cono Sur. Así, brevemente, y sin poder profundizar ahora, apuntaré algunos ejes para el análisis de la relación entre organizaciones indígenas y partidos políticos, así como para identificar experiencias y escenarios de partidos étnicos en México.

\section{La posición zapatista y La Otra política}

En México, la relación entre partidos políticos y organizaciones sociales se ha caracterizado por la posición de clientes en la que éstas han sido colocadas, lo que ha sido un motivo de tensión y de reclamo (Burguete, 2000). Más recientemente, esta relación ha entrado en una fase de confrontación. Como bien se sabe, porque el subcomandante insurgente Marcos ha sido enfático en el deslinde, el Ejército Zapatista de Liberación Nacional (EZLN) desechó la vía político-electoral y repudió cualquier vínculo con partidos políticos, como parte de su estrategia política. Esta definición ha sido más claramente manifiesta desde "La Otra", entendiendo ésta no sólo como "La Otra Campaña" que promovió la comandancia zapatista, sino que también incluye otras iniciativas como "La Otra" (nacional) y la "Zezta internacional", que se integra con varios miles de civiles adherentes en México y en el extranjero. ${ }^{10}$ Por este nuevo perfil, expresamente antiestatal y antielectoral, el EZLN y sus adherentes se han pronunciado por otro tipo de política, distinta a la electoral. Intelectuales adheridos a "La Otra" delínean lo que entienden por la "Otra política"; Aguirre Rojas la define de la siguiente manera:

...mientras la política tradicional gira siempre en torno del momento clímax de las elecciones, y subordina todo a ese momento, apagándose mucho en períodos no electorales y reavivándose enormemente en tiempos de elecciones políticas [...] la Otra Campaña concibe a esta otra política como un asunto de 'todos los días', como una actividad cotidiana y permanente, que se afirma y se despliega en los espacios de trabajo, de vida, de convivencia y de las relaciones sociales más sencillas y elementales... (2006: 89).

Rodríguez, por su parte, apunta:

El defecto fundamental de la política tradicional, sea de derecha o de izquierda, es que concibe la actividad política como algo exclusivo de lo estatal [...] La propuesta zapatista desde el origen, pero más claramente ahora, representa la búsqueda por devolverle a la gente... el monopolio de la decisión política, que parte del presupuesto de que los políticos profesionales (volteando a Maquiavelo) son malos, y que pondrán en práctica sus perversas ideas siempre que se les presente la ocasión de hacerlo deliberadamente (2006: 41-42).

El EZLN se distanció de la vía electoral de manera progresiva, hasta llegar a una ruptura total. Ésta estuvo precedida por una manifiesta decepción de la comandancia y los zapatistas con los partidos políticos, específicamente con el Partido de la Revolución Democrática 
DE ORGANIZACIONES INDÍGENAS A PARTIDOS

ÉTNICOS: NUEVAS TENDENCIAS EN LAS LUCHAS

INDIAS EN AMÉRICA LATINA

Abierta

(PRD). Hernández (2006) menciona que en la "Sexta Declaración de la Selva Lacandona", emitida en junio de 2005, se produjo la crítica pública más profunda del zapatismo a la izquierda institucional, seguida por un largo debate periodístico entre el subcomandante Marcos y distintos dirigentes perredistas e intelectuales de izquierda simpatizantes con ese partido político.

Por ello, por la ruptura epistémica de lo que debe entenderse como "la política" y "lo político" en las estrategias de lucha (con planteamientos muy cercanos a los delineados arriba para los NMS), es muy lejana la posibilidad de que el EZLN, no obstante el alcance nacional de su presencia, se constituya en un "partido étnico", en tanto se diferencia de manera radical del perfil de los movimientos indígenas de Bolivia y Ecuador que transitaron de movimiento social a partido político.

\section{ANIPA: de organización indígena a Agrupación Política Nacional (APN)}

La relación entre los partidos políticos y las organizaciones indígenas ha sido un tema polémico y de polarización entre las organizaciones indígenas mexicanas, en el cual se han privilegiado más las posturas que los debates (Sarmiento, 1999; Flores, 1998; Ruiz, 1999; Burguete, 2000). La Asamblea Nacional Indígena Plural por la Autonomía (ANIPA) nació en 1995 como una organización indígena nacional. Se constituyó con el propósito de impulsar un régimen autonómico para pueblos indígenas y lograr transformaciones que modificaran la configuración monoétnica del Estado y se diera lugar a un Estado multiénico. A diferencia del Congreso Nacional Indígena (CNI) y del EZLN, la ANIPA ha asumido una postura de negociación y alianzas con los partidos políticos para impulsar sus propuestas legislativas, pero también para obtener espacios de representación en la Cámara de Diputados nacional y la de los estados. Las organizaciones que integraron la ANIPA habían convocado, en marzo de 1994, a la Primera Convención Nacional Electoral Indígena en la ciudad de México. A la Asamblea se presentaron todos los candidatos a la presidencia de la República, con excepción del candidato del Partido Revolucionario Institucional (PRI) (Burguete 2000: 162-164). Este diálogo condujo a la negociación de un par de espacios legislativos para diputados indígenas. Volvió a hacer lo mismo para las elecciones intermedias y para las del año 2000 (Ruiz, 1999; Bautista 2000). Con el objeto de lograr un mejor posicionamiento político en la búsqueda de esas alianzas, la ANIPA acordó, en su sexta asamblea celebrada en septiembre de 1996, buscar el registro como Agrupación Política Nacional (APN), el que logró en 1999 (Ruiz, 1999: 44-46; Flores, 2005: 144). ${ }^{11}$

Pese a su tránsito de organización indígena a APN, lo que la puso en el terreno electoral, la ANIPA no ha logrado consolidarse, $y$, por el contrario, en la medida en que el movimiento indígena se debilita, la ANIPA también pierde capacidad de negociación y de incidencia política. En el sistema de partidos en México, dominado por tres fuerzas políticas, las ANP y los partidos políticos pequeños tienen pocas posibilidades de sobrevivencia (Flores, 2005), de lo que resulta muy remoto que la ANIPA pueda constituirse como un "partido político indígena" y menos aún como un "partido étnico". Toda vez que este último requiere un perfil de adhesiones masivas para lograr construir un "movimiento de identificación étnica", con propuestas de carácter universal, con articulaciones multiclasistas, multiétnicas y multisectoriales, entre otros requisitos que se cumplen en Bolivia y Ecuador; y es muy poco probable que todo lo anterior pueda ser cristalizado por el electorado mexicano.

\section{Partido político indígena y procedimiento electo- ral de usos y costumbres en Oaxaca: logros en la ciudadanía política diferenciada}

En un escenario nacional en donde parece lejana la posibilidad de la emergencia de partidos políticos indígenas, al bajar la escala de análisis y explorar en las entidades 
federativas, irrumpe una experiencia: la de Oaxaca. En el mapa de la República mexicana, Oaxaca es pionera al dar nacimiento en julio de 2003 al "primer partido político indígena" (Martínez, 2004): el Partido de Unidad Popular (PUP). El PUP se integró con liderazgos y facciones del Movimiento de Unificación de Lucha Trique (MULT), la Coalición Obrero Campesina Estudiantil del Istmo (COCEI), Nueva Izquierda de Oaxaca (Nioax) y el Frente Oaxaqueño Indígena Binacional (FIOB), entre otras organizaciones de menor tamaño. Este partido contendió, con éxito moderado, en las elecciones municipales para ayuntamientos, diputados y gobernador de esa entidad, en 2004. ${ }^{12}$

En términos de antecedentes, es importante mencionar que años atrás la COCEI había operado como una suerte de partido político indígena en el Istmo oaxaqueño. A pesar de que los emblemas que desplegaba estaban claramente construidos sobre la base de la etnicidad istmeñay su área de influencia iba más allá que la microrregión del Istmo, en los años ochenta, no se asumió expresamente como un "partido político indígena", como sí lo hace ahora el PUP..$^{13}$ Probablemente, la diferencia está dada por el contexto histórico-político en el que ambas experiencias irrumpieron. El PUP nace y se pronuncia como un "partido indígena" en un contexto de politización de la identidad indígena, pero, además, en un marco de reformas legales que reconoce el derecho de los pueblos indígenas a nombrar autoridades mediante sus propias institucionaes (Velásquez, 1999; Anaya, 2003). ${ }^{14}$

Las organizaciones indígenas de Oaxaca han explorado soluciones distintas a las ofrecidas por el sistema electoral de partidos nacional para tratar de resolver la tensión existente entre pueblos indígenas y partidos políticos (Hernández, 1999; Kraemer, 2005; Audelo, 2007). En efecto, hay que recordar que Oaxaca es el único estado que ha legislado para reconocer formas de elegir autoridades municipales distintas a las del sistema político electoral nacional; por ello, la legislación del estado permite el ejercicio de una ciudadanía política diferenciada. Cuenta a su favor con una reforma legislativa que le ha permitido explotar una estrategia de elección de autoridades locales con la que se ha buscado evitar el registro de candidatos municipales mediante los partidos políticos; así, se ha reconocido un mecanismo al que se ha denominado "de usos y costumbres" (Velásquez, 1999; Anaya, 2003).

Pese a la antigüedad e importancia de estas propuestas de reconocimiento de ciudadanía política diferenciada (Hernández, 2007), la experiencia oaxaqueña no se ha diseminado en las distintas entidades federativas del país. De lo que resulta que para la mayoría de los indígenas de México todo se reduce, por ahora, a participar en procesos electorales en el marco del sistema electoral nacional, bajo el monopolio de los partidos políticos.

Pero tal cosa no es circunstancial. Conviene recordar que, en su hipótesis, Van Cott (2003) planteaba que la condición para la irrupción de partidos étnicos era la de los cambios institucionales de reconocimiento de derechos (de ciudadanías políticas diferenciadas, aunque no le llame así) y de apertura del sistema de partidos para simplificar la participación de actores políticos indígenas, que hicieran posible la representación y el autogobierno indígena. Así, en Oaxaca pareciera cumplirse la premisa de la EOP. La irrupción de partidos políticos indígenas y de formas alternas no tiene su explicación en el peso demográfico indígena (sin que se ignore su importancia), sino que es resultado de las condiciones creadas a partir de los cambios institucionales, que abren oportunidades e incentivan a los actores políticos a explorar otras vías de representación y la irrupción de nuevos actores políticos demandantes, que reclaman la ocupación de esos espacios.

A diferencia de Oaxaca, en el resto de México las reglas de la democracia electoral son la única vía legal para la participación política indígena, toda vez que desde 1996 ésta ha sido la única oferta política que el sistema político ha ofrecido a los pueblos indígenas después de haber ignorado la reglamentación del Convenio 169 de la Organización Internacional del Trabajo, en la legislación 
DE ORGANIZACIONES INDÍGENAS A PARTIDOS

nacional, e incumplidos los Acuerdos de San Andrés. De esta forma, ha negado el reconocimiento y la realización de derechos de ciudadanías políticas diferenciadas.

Recordemos que en el "Documento 3.2. Acciones y Medidas para Chiapas. Compromisos y propuestas conjuntas de los gobiernos del estado y federal y el EZLN" se estableció lo siguiente:

Esta reforma deberá garantizar condiciones de mayor equidad y transparencia en los procesos electorales, reconocer el derecho de las comunidades para nombrar a sus autoridades tradicionales y municipales de acuerdo a sus usos y costumbres, y otorgar validez jurídica a las instituciones y prácticas de las comunidades indígenas para nombrar autoridades y realizar consultas bajo esquemas incluyentes y sin la necesaria participación de los partidos políticos (Hernández y Vera, 1998: 87).

Como sabemos, los Acuerdos de San Andrés siguen siendo una deuda que debe cumplirse desde el Estado, el que deberá promover, entre otras cosas, con base en dichos acuerdos, reformas institucionales y modificar el sistema de partidos políticos. En un escenario de su probable realización, en algún momento dado, las reformas institucionales podrían abrir oportunidades para la representación política, el autogobierno y el fortalecimiento de las instituciones de representación y elección de autoridades de los pueblos indígenas en México.

\section{A modo de reflexión final}

Desde su nacimiento en la década de los setenta (Stavenhagen, 1997), las organizaciones indígenas en América Latina han estado en permanente transformación, renovándose continuamente, en una dialéctica en la que con sus luchas transforman el Estado y la sociedad; al mismo tiempo que son transformadas por éstos en una lógica de constitución mutua. Habiendo irrumpido desde el seno de las luchas campesinas y campesinistas, se deslindaron de ellas en los años sesenta y setenta. Pos- teriormente, se reconfiguraron para constituirse en organizaciones indígenas comunitarias, locales, dando un paso fundacional, al configurarse como un movimiento indígena de carácter pantétnico, de alcance translocal, en los ámbitos nacional e internacional, en un contexto generalizado de emergencia indígena (Bengoa, 2000; Gros, 2000). Hoy, asistimos a una reconfiguración de las organizaciones indígenas latinoamericanas, las que se han estado constituyendo en partidos políticos.

Esta tendencia se ha originado en el cono sur, en Ecuador, Bolivia, Colombia, Venezuela y Argentina. Pero no se agota allí. En otros países como Chile, Perú y Guatemala, las organizaciones crean partidos políticos indígenas, aun cuando sus probabilidades de éxito sean remotas, como ha sido previsible en el caso de Guatemala. Sin embargo, esa estrategia busca llamar la atención del Estado, de los actores políticos, de la sociedad civil, nacional e internacional, para demandar reformas en el sistema electoral, en el sistema de partidos políticos, para que éste se transforme y crear, de esta manera, condiciones para ampliar la representación ciudadana, y reconocer, así, la ciudadanía política diferenciada. El "ejemplo Evo Morales" ha contribuido a alentar esta tendencia.

\section{Notas}

${ }^{1}$ Cfr. Primer Encuentro Continental de Pueblos y Nacionalidades Indígenas de Abya Yala: De la resistencia al poder", La Paz, Bolivia, octubre de 2006, y "III Cumbre Continental de Pueblos y Nacionalidades Indígenas de Abya Yala: De la resistencia al poder", Guatemala, mazo de 2007.

${ }^{2}$ Cfr. "Guatemala: Indígenas y campesinos conformarán nuevo partido político", 18 de diciembre de 2006, http:// www.radiomundoreal.fm/rmr/?q=es/node/20811Cfr. "Guatemala: Indígenas y campesinos conformarán nuevo partido político", 18 de diciembre de 2006, http:// www.radiomundoreal.fm/rmr/?q=es/node/2081l. Hernández Pico, Juan (s/f), Guatemala ¿Puede ocurrir, Evo Morales, en Guatemala?", http://www.envio.org. ni/articulo/3336. "Movimiento indígena de Guatema- 
la pretende crear un partido político", Los tiempos. com internacional, 17 de diciembre 2006, http:/www. lostiempos.com/noticias/17-12-06/17_12_06_inter5.php. Editorial ¿Es un "partido indígena" la solución? http:// www.cisle.org.mx/editorial/indigena.htm.

3 "Perú. Nace el Movimiento al Socialismo Andino Amazónico", Regiones Indias 8 de mayo de 2007, versión digital, Agencia Internacional de Prensa India (AIPIN), México. 4 "Partido Originario Mapuche hace su estreno en Zapala", Regiones Indias 23 de mayo de 2007, versión digital, Agencia Internacional de Prensa India (AIPIN), México. ${ }^{5}$ El archivo completo del proceso puede verse en la página web del candidato, Cfr., http://www.mapuche-nation. org/espanol/html/acciones/aucan_presidente.htm

${ }^{6}$ El llamado Consenso de Washington se estableció en 1989 como una agenda para los países llamados "del sur o "en vías de desarrollo" y contienen 10 puntos: 1)Disciplina fiscal. 2)Gasto en educación y salud. 3)Reforma tributaria. 4)Tasas de interés positivas determinadas por el mercado. 5)Tipos de cambio competitivos. 6) Políticas comerciales liberales. 7)Mayor apertura a la inversión extranjera. 8)Privatización de empresas públicas. 9) Desregulación. 10)Protección a la propiedad privada.

${ }^{7}$ La Teoría de la Elección Racional fue formulada a partir de la obra de M. Olson, The Logic of Collective Action (1965), en la que se plantea que los individuos participan en la acción colectiva utilizando un razonamiento estratégico e instrumental, basado en el cálculo en función de sus intereses y tras un cálculo de los costes y beneficios que les supone dicha participación, es decir, el coste nunca puede ser mayor que el beneficio que se espera conseguir. Además, se observa la movilización como un problema de "gestión de recursos" (resource management). La movilización alude a los procesos por los que un grupo descontento reúne e invierte recursos para conseguir los objetivos del grupo. La racionalidad de los actores de la protesta es evidente ya que ellos sopesan las recompensas y sanciones, costes y beneficios, que los cursos de acción alternativos representan para ellos (Rubio, 2004; Cohen, 1988; Jenkin, 1988).
${ }^{8}$ Impulsado por Menchú y otros reconocidos líderes indígenas, crearon el "Movimiento Winaq" con el propósito de sumar a otras organizaciones indígenas, principalmente las que se adscriben al movimiento maya. Sin embargo, por razones de legislación electoral, aunado a la poca aceptación desde las organizaciones indígenas, y por la indefinición política de Menchú, el proyecto del partido étnico no prosperó. Sobre este punto ver el análisis de Ba Tiul (2007).

${ }^{9}$ La "Otra campaña" consistió en una gira realizada por el subcomandante Marcos para entrar en contacto con diversos grupos en distintas partes del país. Cfr. http://enlacezapatista.ezln.org.mx/category/la-otra-campana/

${ }^{10}$ En la red electrónica se encuentran numerosas páginas sobre los colectivos de "La Otra", en distintas ciudades de México y el extranjero. Cfr. http:/www.zeztainternazional.org/; http://cml.vientos.info/node/1832.

11 "La Agrupación Política Nacional es una forma de asociación ciudadana cuyo propósito es contribuir a crear las condiciones para el desarrollo de la vida democrática y la creación de cultura política y opinión pública bien informada en el país. La base jurídica de la Agrupación Política Nacional son los artículos 33 al 35 del Capítulo Segundo del Código Federal y Procedimierntos Electorales (COFIPE)" (Ruiz, 1999: 46).

${ }^{12}$ Notas periodísticas diversas informaron que en julio de 2003, cerca de 10 mil indígenas triquis, mixtecos y zapotecos decidieron constituir el Partido de la Unidad Popular (PUP) con miras a participar en el proceso electoral local de 2004, en el que se elegiría gobernador, diputados locales y presidentes municipales, en Oaxaca. El PUP obtuvo su registro en noviembre de ese mismo año. Los resultados fueron inferiores a la expectativa. No ganó ningún municipio y obtuvo solamente una diputación. Aunque sus líderes eran viejos militantes y dirigentes del Partido de la Revolución Democrática (PRD) y de organizaciones sociales indígenas de larga data, no logró distraer el "voto duro" de ese partido, ya que fueron acremente cuestionados, acusados de manejar alianzas y agendas ocultas. No 
DE ORGANIZACIONES INDÍGENAS A PARTIDOS

obstante el poco respaldo social a la iniciativa, generó mermas suficientes que abonaron a favor del triunfo del actual gobernador Ulises Ruiz, según fuentes periodísticas. Algunas de las figuras más visibles de ese partido son Flavio Sosa, líder de la Asamblea Popular del Pueblo de Oaxaca (APPO), actualmente en la cárcel, y Héctor Sánchez de la COCEI. Cfr. "Campesinos oaxaqueños fundan partido, aun sin aval del IEE. El PUP surge de la fusión de tres movimientos; obtener el registro, el reto, señala su comité", Diario La Jornada, México D.F. Miércoles 30 de julio de 2003. "Crean en Oaxaca partido político indígena. Cerca de 10 mil triquis, mixtecos y zapotecos se aglutinan en el PUP. Buscan el registro ante el IEE para contender en las elecciones locales del 2004", Pedro Matías (APRO), 30 de julio de 2003, http://www.nciv.net/spaans/noticia03.htm.

${ }^{13}$ Giménez (1994) caracterizó a la COCEI como un "partido político local"; mientras que Campbell (1989) destacó la importancia de la etnicidad en la configuración de la COCEI y sus estrategias políticas partidarias.

${ }^{14}$ Para Tarrow (2004), éste sería un ejemplo muy claro que explicaría el éxito de algunas organizaciones o movimientos indígenas, favorecidos por una "Estructura de Oportunidades Políticas". En este caso, la legislación oaxaqueña que reconoce el ejercicio de derechos de ciudadanía política diferenciada (Hernández, 2007).

\section{Bibliografía}

Aguirre Rojas, Carlos A., 2006, “La ‘Otra política’ de La otra Campaña: la muerte de la política y el renacimiento del poder social", en Contrahistorias. La otra mirada de Clío, núm. 6, marzo-agosto, México, pp. 81-92.

Albó, Xavier, 1996, "Nación de muchas naciones: nuevas corrientes políticas en Bolivia", en Pablo González Casanova y Marcos Roitman Rosenmann (cords.), Democracia y Estado multiétnico en América Latina, Centro de Investigaciones Interdisciplinarias en Ciencias y Humanidades, UNAM, Demos, México, pp. 321-366.
Anaya Muñoz, Alejandro, 2003, "La política del reconocimiento en Oaxaca: la preservación de una gobernabilidad priísta y el desarrollo del proyecto de autonomía indígena en el estado", en Relaciones, otoño, año/vol. XXIV, núm. 096, Zamora, Colegio de Michoacán, Michoacán, pp. 267-304.

Audelo Cruz, Jorge Mario, 2007, "Organizaciones sociales y partidos políticos en Oaxaca: sus vínculos”, en Política y Cultura, núm. 27, primavera, UAM-X, México, pp. 57-74.

Bautista, Genaro, 2000, "El acercamiento con los presidenciables”, en Palabra India, núm. 2, Año 1, ANIPA, , México, pp. 9-11.

Ba Tiul, Kajkoj Maximo, 2007, "Movimiento Winaq, la controversia: ni a la izquierda ni a la derecha", documento de trabajo del seminario "Izquierdas y construcción de orden democrático en Guatemala”, organizado por FLACSO Guatemala y la Fundación Friedrich Ebert, borrador para la discusión, Junio de 2007, www.albedrio.org/htm/documentos/winaqMaxBaTiul.pdf.

Barrera, Augusto, 2001, Acción colectiva y crisis política. El movimiento indígena ecuatoriano en la década de los noventa, Abya Yala, Quito, Ecuador.

Bengoa, José, 2000, La emergencia indígena en América Latina, FCE, México.

Boron, Atilio, 2005, "El dilema de los movimientos sociales: a organizar la desorganización”, en Rebelión. Construyendo poder desde abajo, 14.07.2005.http://www. rebelion.org/noticia.php?id=17776.

—, 2006, "Crisis de las democracias y movimientos sociales en América Latina: notas para una discusión”. Rebelión. Construyendo poder desde abajo, 16.12.2006, http:// www.rebelion.org/noticia.php?id=43203.

Botero Villegas, Luis Fernando, 1998, "Estado, cuestión agraria y movilización india en Ecuador. Los desafíos de la democracia", en Nueva Sociedad, núm. 153, enero-febrero, Nueva Sociedad, Buenos Aires, Argentina, pp. 56-72.

Burguete Cal y Mayor, Araceli, 2000a, "Ecuador 2000: la primera rebelión indígena del tercer milenio", en Memoria, núm. 133, marzo, CEMOS, pp. 31-35, México. 
—, 2000b, "Partidos políticos y pueblos indígenas en México: crónica de desencuentros", en Natividad Gutiérrez Chong, Marcela Romero y Sergio Sarmiento, (coordinadores) Indigenismos. Reflexiones críticas, INI, México, pp. 145-177.

Calla Ortega, Ricardo, 1993, "Hallu hayllisa huti. Identificación étnica y procesos políticos en Bolivia (1973-1991)”, en Alberto Adriazén et al., Jornadas de Politología. Democracia, etnicidad y violencia política en los países andinos, IEP, IFEA, Lima, Perú.

Campbell, Howard B., 1989, "La COCEI: cultura y etnicidad politizadas en el istmo de Tehuantepec", en Revista Mexicana de Sociología, año LI, núm. 2, abril-junio, IIS, UNAM, México, pp. 247-263.

Carvajal A., Miguel, 2004, "Pachakutik: la efímera experiencia de gobierno y las incógnitas sobre su futuro", en Íconos. Revista de Ciencias Sociales, enero, núm. 018, FLACSO-Ecuador, Quito, Ecuador, pp. 6-9.

Cohen, Jean, 1988, "Estrategia o identidad: paradigmas teóricos nuevos y movimientos sociales contemporáneos", en FLACSO (comp.), Teoría de los movimientos sociales, FLACSO, Costa Rica, pp. 3-44.

Cheresky, Isidoro, 2006, "Elecciones en América Latina: poder presidencial y liderazgo político bajo la presión de la movilización de la opinión pública y la ciudadanía”, en Nueva Sociedad, núm. 206, noviembre-diciembre, Nueva Sociedad, Buenos Aires, Argentina, pp. 14-26.

Dávalos, Pablo, 2003, Plurinacionalidad y poder político en el movimiento indígena ecuatoriano, en OSAL, enero, Buenos Aires, Argentina.

Díaz-Polanco, Héctor y Consuelo Sánchez, 2002, México diverso: el debate por la autonomía, Siglo XXI, México.

Flores Andrade, Anselmo, 2005, "Democracia y dinero. Partidos nuevos y asociaciones políticas nacionales en México", en Reflexión política, núm. 13, Año/Vol. 7, junio, Universidad Autónoma de Bucaramanga, Colombia, pp. 134-153.

Flores Félix, José Joaquín, 1998, La revuelta por la democracia. Pueblos indios, politicay poder en México, UAM, El Atajo Ediciones, México.
García, Fernando, 2005, De movimiento social a partido político: el caso del Movimiento de Unidad Plurinacional Pachakutik-Ecuador, ponencia presentada en el "Seminario Partidos de izquierda y movimientos sociales en América Latina" realizado del 31 de octubre al 2 de noviembre de 2005, en Sao Paulo-Brasil, por la Fundación Rosa Luxemburgo http://www.rls. org.br/publique/media/PartAL_Garcia.pdf.

García Linera, Álvaro, 2005, “Los movimientos indígenas en Bolivia”, en Fabiola Escárzaga y Raquel Gutiérrez (coords.), Movimiento indígena en América Latina: resistencia y proyecto alternativo, vol. I, Casa Juan Pablos y coeditores, México, pp. 76-84.

Giménez, Gilberto, 1994, "Comunidades primordiales y modernización en México", en Gilberto Jiménez y Ricardo Pozas (coordinadores), Modernización e identidades sociales, UNAM, México, pp. 149-183.

González Casanova, Pablo, 1993, "La democracia de los de abajo y los movimientos sociales", en Memoria, núm. 54, CEMOS-Memoria, México, pp. 20-22.

Guerrero, Andrés, 1996, "El levantamiento indígena de 1994. Discurso y representación política en Ecuador”, en Revista Nueva Sociedad, núm. 142, marzo-abril, Buenos Aires, Argentina, pp. 32-43.

Gros, Christian, 2000, "Ser diferente para ser moderno, o las paradojas de la identidad. Algunas reflexiones sobre la construcción de una nueva frontera étnica en América Latina", en Leticia Reina (coordinadora), Los retos de la etnicidaden los estados-nación del siglo XXI, CIESAS, INI, Miguel Ángel Porrúa Editores, México, pp. 171-196.

Hernández Castillo, Aída, 2006, "The Indigenous Movement in Mexico: Between Electoral Politics and Local Resistance", en Latin American Perspectives 33, MarchApril, USA, pp. 115-131.

Hernández Díaz, Jorge, 2007, "Dilemas en la construcción de ciudadanías diferencias en un espacio multicultural: el caso de Oaxaca", en Jorge Hernández Díaz (coord.), Ciudadanías diferenciadas en un estado multicultural. Los usos y costumbres en Oaxaca, Siglo XXI, UABJO, México, pp. 35-86. 
DE ORGANIZACIONES INDÍGENAS A PARTIDOS

—, 1999, "Las organizaciones indígenas y su participación electoral en Oaxaca", en Trace, núm. 36, diciembre, CEMCA, México, pp. 76-84.

Hernández Navarro, Luis y Ramón Vera (comp.), 1998, Acuerdos de San Andrés, ERA, México.

Hidalgo Flor, Francisco, 2002, "Elecciones en Ecuador: quiebra de los partidos políticos y la presencia del movimiento indígena", en Revista de Ciencias Sociales, año/vol. IV, núm. 098, Universidad de Costa Rica, San José, Costa Rica, pp. 87-97.

Jenkins, Craig, 1988, "La teoría de la Movilización de Recursos y el estudio de los movimientos sociales", en FLACSO (comp.), Teoría de los movimientos sociales, FLACSO, Costa Rica, pp. 45-69.

Kraemer Bayer, Gabriela, "Los partidos políticos y la autonomía en la región mixe, Oaxaca”, en Gabriela Kraemer y Martha Judit Sánchez (coords.), De derechos étnicos y territoriales de la población indígena y campesina en México, AMER, Editorial Praxis, México, pp. 199-228.

Máiz, Ramón, 2005, "El indigenismo político en América Latina", en Jornadas Pueblos Indígenas de América Latina, Panel l: Visibilidad de los pueblos indígenas, Obra social. Fundación La Caxia, Programa de Cooperación Internacional, Barcelona, 27 y 28 de abril, http://portall.lacaixa.es/Docs/Chan/95/pdf/01_pl_ maiz.pdf.

Mamani Ramírez, Pablo, 2006, "Dominación étnica, de clase y territorialización del poder indígena en Bolivia”, en Raquel Gutiérrez y Fabiola Escárzaga (coords.), Movimiento indígena en América Latina: resistencia y proyecto alternativo, vol. II, Casa Juan Pablos y coeditores, México, pp. 35-53.

Martí i Puig, 2006, Un estudio prospectivo sobre la presencia y la relevancia de los partidos indigenistas en América Latina, documentos CIDOB, núm. 10, Barcelona, Fundación CIDOB, Ediciones.

http://www.cidob.org/es/publicaciones/documentos_cidob/america_latina/un_estudio_prospectivo_sobre la_presencia_y_la_relevancia_de_los_partidos_indigenistas_en_america_latina.
—, 2004, Sobre la emergencia y el impacto de los movimientos indígenas en las arenas políticas de A mérica Latina, Documentos CIDOB, núm. 2, Barcelona, Fundación CIDOB Ediciones.

http://campus.usal.es/ dpublico/areacp/Doctorado0304/ Seminario_Investigacion03/Smarti04.PDF.

Martínez, Sánchez Francisco, 2004, "El primer partido político indígena en México”, en Revista Derecho y Cultura, núm. 13, enero-abril, IIJ, UNAM, México, pp. 103-116.

Mayorga, René Antonio, 2003, "Bolivia: metamorfosis del sistema de partidos”, en Íconos, Revista de Ciencias Sociales, mayo, número 016, FLACSO, Quito, Ecuador, pp. 96-105.

Melucci, Alberto, 1999, Acción colectiva, vida cotidiana y democracia, El Colegio de México, México.

Mirza, Christian Adel, 2006, Movimientos sociales y sistemas políticos en América Latina: la construcción de nuevas democracias, CLACSO, Buenos Aires, Argentina.

Offe, Claus, 1998, Partidos políticos y nuevos movimientos sociales, Editorial Sistema, Madrid, España.

Oñate, Pablo, 2005, "Participación política, partidos y nuevos movimientos sociales", en Revista Mexicana de Ciencias Políticas y Sociales, mayo-agosto, año/vol, XLVII, núm. 194, UNAM, México, pp. 103-135.

Patzi Paco, Félix, 2005, "Las tendencias en el movimiento indígena en Bolivia”, en Fabiola Escárzaga y Raquel Gutiérrez (coords.), Movimiento indígena en América Latina: resistencia y proyecto alternativo, vol. I, Casa Juan $\mathrm{Pa}$ blos y coeditores, México, pp. 68-74.

Quispe, Felipe, 2006, "Dos repúblicas en Bolivia", en Raquel Gutiérrez y Fabiola Escárzaga (coords.), Movimiento indígena en América Latina: resistencia y proyecto alternativo, vol. II, Casa Juan Pablos y coeditores, México, pp. 54-59.

Ramos Rollón, María Luisa, 1997, "La dimensión política de los movimientos sociales: algunos problemas conceptuales", en Revista Española de Investigaciones Sociológicas, núm. 79, julio-septiembre, Centro de Investigaciones Sociológicas, Universidad Pública de Navarra, España, pp. 247-263. 
Rivera Cusicanqui, Silvia, 2003, Oprimidos pero no vencidos. Luchas del cammpesinado aymara y qhechwa 1900-1980, Aruwiyiri-Editorial del Taller de Historia Oral Andino (THOA), tercera edición, La Paz, Bolivia.

Rodríguez Lazcano, Sergio, 2006, "Once tesis y una premonición sobre la otra política zapatista”, en Contrahistorias, núm. 6, marzo-agosto, La otra mirada de Clío, México, pp. 41-48.

Rubio García, Ana, 2004, "Perspectivas teóricas en el estudio de los movimientos sociales", en Circunstancia Año 1, núm.3, enero, Revista Electrónica de Ciencias Sociales del Instituto Universitario de Investigación Ortega y Gasset, España. http://www.ortegaygasset.edu/circunstancia/numero3/art4.htm.

Ruiz Hernández, Margarito y Araceli Burguete Cal y Mayor, 2001, "Indigenous Peoples without Political Parties: The dilemma of Indigenous Representation in Latin America", en Kathrin Wessendorf (editor), Challengin Politics: Indigenous Peoples. Experiences with Political Parties and Elections, IWGIA, Copenhagen, pp. 20-62.

Ruiz Hernández, Margarito, 1999, "La Asamblea Nacional Indígena Plural por la Autonomía (ANIPA). Proceso de construcción de una propuesta legislativa autonómica nacional", en Araceli Burguete Cal y Mayor (coord.), México: experiencias de autonomía indígena, IWGIA, Guatemala, pp. 21-53.

Ruiz Murrieta, Julio, 2003, Democraciay participación política de lospueblosindígenasenAméricaLatina,París,UNESCO,http:// unesdoc.unesco.org/images/0012/001297/129780s.pdf.

Santos, Boaventura Sousa, 2001, Los nuevos movimientos sociales, OSAL, Buenos Aires, Argentina,

http://168.96.200.17/ar/libros/osal/osal5/debates.pdf.

Sarmiento, Sergio, 1999, "Pueblos indios, partidos políticos y procesos electorales”, en Trace, núm. 36, diciembre, CEMCA, México, pp. 51-56.

Somuano Ventura, Ma. Fernanda, 2007, "Movimientos sociales y partidos políticos en América Latina: relación cambiante y compleja", en Política y Cultura, núm. 27, primavera, UAM-X, México, pp. 31-53.
Stavenhagen, Rodolfo, 1997, "Las organizaciones indígenas: actores emergentes en América Latina”, en Revista de la CEPAL, núm. 62, agosto, Santiago de Chile, CEPAL, ONU, pp. 61-73.

Tarrow, Sydney, 2004, El poder en movimiento. Los movimientos sociales, la acción colectiva y la política, Alianza, Madrid, España.

Touraine, Alain, 2006, "Entre Bachelet y Morales, iexiste una izquierda en América Latina?”, en Nueva Sociedad, núm. 205, septiembre-octubre, Nueva Sociedad, Buenos Aires, Argentina, pp. 46-55.

Van Cott, Donna Lee, 2005, From Movements to Parties in Latin American: the evolution of ethnic politics, Cambridge, Cambridge University Press.

—, 2004, "Los movimientos indígenas y sus logros: la representación y el reconocimiento jurídico en los Andes", en América Latina Hoy, abril, núm. 036, Salamanca, España, Universidad de Salamanca, pp. 141-159.

—, 2003, "Cambio institucional. Partidos étnicos en Sudamerica”, en Análisis Político, núm. 48, enero-abril, Universidad Nacional de Colombia, Bogotá, pp. 26-51.

Velásquez, Cepeda, María Cristina, 1999, "Fronteras de gobernabilidad municipal en Oaxaca, México: el reconocimiento jurídico de los 'usos y costumbres' en la renovación de los ayuntamientos indígenas", en Willem Assies, Gemma van der Haar y André Hoekema (editores), El reto de la diversidad. Pueblos indígenas y reforma del Estado en América Latina, México, COLMICH, Zamora, Michoacán, pp. 289-314.

\section{Fuentes primarias}

"Campesinos oaxaqueños fundan partido, aun sin aval del IEE. El PUP surge de la fusión de tres movimientos; obtener el registro, el reto, señala su comité", Diario La Jornada, México D.F., Miércoles 30 de julio de 2003,

http://www.jornada.unam.mx/2003/07/30/034flest. php?origen=estados.php\&efly=2.

"Crean en Oaxaca partido político indígena. Cerca de 10 mil triquis, mixtecos y zapotecos se aglutinan en 
el PUP. Buscan el registro ante el IEE para contender en las elecciones locales del 2004", Pedro Matías (APRO), 30 de julio de 2003, http://www.nciv.net/ spaans/noticia03.htm.

"Guatemala: Indígenas y campesinos conformarán nuevo partido político", 18 de diciembre de 2006, http:// www.radiomundoreal.fm/rmr/?q=es/node/2081l.
Hernández Pico, Juan (s/f), Guatemala ¿Puede ocurrir "Evo Morales", en Guatemala? http://www.envio.org. ni/articulo/3336;

"Movimiento indígena de Guatemala pretende crear un partido político", Los tiempos.com internacional, 17 de diciembre 2006, http://www.lostiempos.com/noticias/17-12-06/17_12_06_inter5.php. 